\title{
OCCUPATIONAL HEALTH CARE PROVIDED TO WORKERS EXPOSED TO HEAT RELATED INDUSTRIES
}

\author{
By \\ Helal S \\ Department of Occupational and Environmental Medicine \\ Faculty of Medicine - Cairo University
}

\begin{abstract}
:
The temperature of the environment is one of the factors affecting human performance. At body temperature substantially higher than the optimal levels $\left(36.5-37.5^{\circ} \mathrm{C}\right)$, both physical and mental performance may deteriorate due to the complicated interplay of physiological and pathophysiological processes. The main source of heat gain is the body's own internal heat. When the air temperature or humidity rises above the optimal ranges for comfort, problems can arise. From these problems: heat stroke, heat exhaustion, heat syncope, heat cramps, skin rash and fainting. Transient heat fatigue refers to the temporary state of discomfort and mental or psychological strain arising from prolonged heat exposure. The risk of heat stress arises from a combination of human physical effort, which increases body temperature, and the ability of the body to shed the excess heat in order to maintain a viable core body temperature. Heat loss from the body depends on environmental factors such as radiant heat, air temperature, relative humidity and air movement. Heat control measures include: (1) Engineering controls to provide a cooler workplace. (2) Personal Protection to reduce worker exposure. (3) Training employees to recognize and prevent heat illnesses (Health education).
\end{abstract}

Key Words: Heat stress- Heat gain- Heat loss-Convection-Conduction-Radiation. 


\section{Introduction}

The World Health Organization (WHO) defines occupational health as a multidisciplinary activity that promotes and protects the health of workers. This discipline seeks to control accidents and diseases by reducing risk conditions.

Occupational health care is not limited to physical conditions of the worker, but also addresses the psychological question. For employers, occupational health support is a refinement of the worker and the maintenance of working capacity (ACGIH, 2006).

The problems of work in extreme environments have been studied for many years. The ability to perform physical work depends on the ability of the muscle cell to transform chemically bound energy in the food which we eat into mechanical energy for muscular work. This in turn depends on the capacity of the service functions that deliver fuel and oxygen to the working muscle fibre (Rodahl, 2003).

The working technique may be of major importance in conserving energy and in providing varied use of different muscle groups. The monotony of a working operation may be a stress for some individuals but a relief for others who can carry on the work more or less automatically while thinking about something else. In any case, the tempo of work performance may be extremely important and impose stresses which, in some instances, may be unbearable or harmful to the individual (ACGIH, 2006).

From these industries: Outdoor operations in hot weather, including surface mining, roofing, road repair and construction, dam building, and other construction ,farming operations ,iron, steel and nonferrous foundries ,brick-firing and ceramics operations, glass products manufacturing plants ,rubber products manufacturing plants ,electrical utilities (particularly boiler rooms) ,bakeries, confectioneries ,restaurant kitchens ,laundries ,food canneries ,chemical manufacturing facilities ,mines, smelters , steam tunnels.

The temperature of the environment is one of the factors affecting human performance. At body temperatures substantially higher than the optimal levels $\left(36.5-37.5^{\circ} \mathrm{C}\right)$, both physical and mental performance may deteriorate due to the complicated interplay of physiological and pathophysiological processes. Prolonged heat stress may lead to loss of body fluid (hypohydration), which in itself impairs performance, especially endurance. In addition, prolonged heat strain may impair 
mental and psychomotor functions, thereby affecting performance. It is, therefore, of considerable practical importance to be able to assess the magnitude of the thermal stress in the working environment and the worker's physiological reaction to it, in order to ensure optimal conditions for health and productivity (Harrington, 2001).

\section{How does the human body react to}

\section{hot environments?}

The healthy human body maintains its internal temperature around $37^{\circ} \mathrm{C}$. Variations, usually of less than $1^{\circ} \mathrm{C}$, occur with the time of the day, level of physical activity or emotional state. A change of body temperature exceeding $1^{\circ} \mathrm{C}$ occurs only during illness or when environmental conditions surpass the body's ability to cope with extreme temperatures.

As the environment warms-up, the body tends to warm-up as well. The body's internal "thermostat" maintains a constant inner body temperature by pumping more blood to the skin and by increasing sweat production. In this way, the body increases the rate of heat loss to balance the heat burden created by the environment. In a very hot environment, the rate of "heat gain" exceeds the rate of "heat loss" and the body temperature begins to rise. A rise in the body temperature results in heat illnesses (OSHA, 2010).

\section{How does the body control heat gain and heat loss?}

The main source of heat gain is the body's own internal heat.; called metabolic heat, it is generated within the body by the biochemical processes that keep us alive and by the energy we use in physical activity. The body exchanges heat with its surroundings mainly through radiation, convection, and evaporation of sweat.

Radiation is the process by which the body gains heat from surrounding hot objects, such as hot metal, furnaces or steam pipes, and loses heat to cold objects, such as chilled metallic surfaces, without contact with them. No radiant heat gain or loss occurs when the temperature of surrounding objects is the same as the skin temperature (about $35^{\circ} \mathrm{C}$ ).

Convection is the process by which the body exchanges heat with the surrounding air. The body gains heat from hot air and loses heat to cold air which comes in contact with the skin. Convective heat exchange increases with increasing air speed and increased differences between air and skin temperature.

Evaporation of sweat from the skin cools the body. Evaporation proceeds more quickly and the cooling effect is more pronounced with high wind speeds and 
low relative humidity. In hot and humid workplaces, the cooling of the body due to sweat evaporation is limited by the capacity of the ambient air to accept additional moisture. In hot and dry workplaces, the cooling due to sweat evaporation is limited by the amount of sweat produced by the body.

The body also exchanges small amounts of heat by conduction and breathing. By conduction, the body gains or loses heat when it comes into direct contact with hot or cold objects. Breathing exchanges heat because the respiratory system warms the inhaled air. When exhaled, this warmed air carries away some of the body's heat. However, the amount of heat exchanged through conduction and breathing is normally small enough to be ignored in assessing the heat load on the body (NIOSH, 2010).

\section{What are the effects of hot environments on the body?}

When the air temperature or humidity rises above the optimal ranges for comfort, problems can arise. The first effects are subjective in nature - they relate to how you feel. Exposure to more heat stress can cause physical problems which impair workers' efficiency and may cause adverse health effects. (Short-term exposure / Long-term exposure).

Some of the problems and their symptoms experienced in the temperature range between a comfortable zone $(20 \mathrm{C}$ $27^{\circ} \mathrm{C}$ ) and the highest tolerable limits (for most people) are summarized in Table 1. 
Table 1

Problems and Symptoms Caused by Hot Temperatures

\begin{tabular}{|c|c|c|}
\hline Temperature Range $\left({ }^{\circ} \mathrm{C}\right)$ & \multicolumn{2}{|l|}{ Effects } \\
\hline $20-27^{\circ} \mathrm{C}$ & Comfort Zone & Maximum efficiency \\
\hline \multirow[t]{3}{*}{ as temperature increases... } & 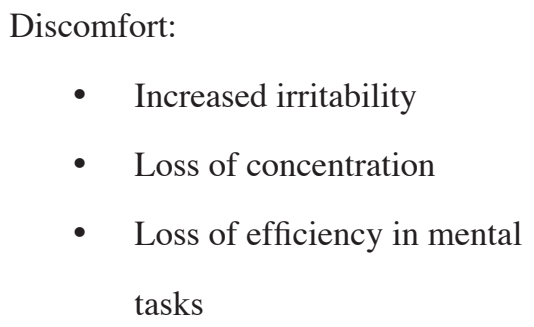 & Mental Problems \\
\hline & 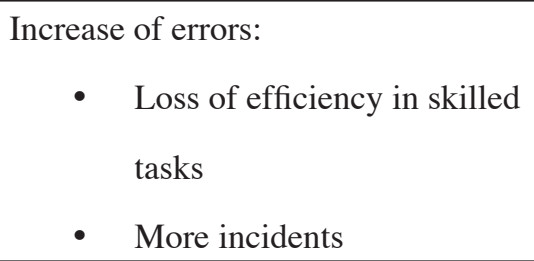 & $\begin{array}{l}\text { Pyscho-physiological } \\
\text { problems }\end{array}$ \\
\hline & $\begin{array}{l}\text { Loss of performance of heavy work: } \\
\text { - } \quad \text { Disturbed water and } \\
\text { electrolyte balance } \\
\text { - } \quad \text { Heavy load on heart and } \\
\text { circulation } \\
\text { - Fatigue and threat of } \\
\text { exhaustion }\end{array}$ & Physiological problems \\
\hline $35-40^{\circ} \mathrm{C}$ & \multicolumn{2}{|l|}{ Limit of high temperature tolerance } \\
\hline
\end{tabular}

(NIOSH, 2010a) 


\section{The effect of heat stress on heart rate}

It is well known that heat stress may represent an additional load on the cardiovascular system. This is evidenced by an elevated heart rate at the same work load in a hot environment versus a room temperature environment. The explanation is that, in the case of the heat stress, our circulating blood volume, in addition to having to transport oxygen, also has to serve as a cooling fluid. It therefore transports heat from the interior of the body to the skin where it is dissipated to the surrounding environment by conduction, convection, radiation and sweat evaporation. This requires an increase in speed of the blood circulation, i.e. the cardiac output (minute volume) has to be elevated. This can only be done by increasing the stroke volume of the heart and/or increasing the heart rate. Since the possibility of increasing the stroke volume of the heart is limited, a major increase in the minute volume can only be achieved by an increase in the heart rate. Thus, the heart rate becomes an expression of the magnitude of the additional load exerted on the cardiovascular system when the body is exposed to a certain heat stress. It is, therefore, to be expected that the heart rate increases with increasing body temperature. In turn, this is affected by the temperature of the environment as well as the work rate ( Rodahl , 2003).
Workers who are exposed to extreme heat or work in hot environments may be at risk of heat stress. Exposure to extreme heat can result in occupational illnesses and injuries. Heat stress can result in heat stroke, heat exhaustion, heat cramps, or heat rashes. Heat can also increase the risk of injuries in workers as it may result in sweaty palms, fogged-up safety glasses, and dizziness. Burns may also occur as a result of accidental contact with hot surfaces or steam (5). Workers at risk of heat stress include outdoor workers and workers in hot environments such as firefighters, bakery workers, farmers, construction workers, miners, boiler room workers, factory workers, and others. Workers at greater risk of heat stress include those who are 65 years of age or older, are overweight, have heart disease or high blood pressure, or take medications that may be affected by extreme heat.

Prevention of heat stress in workers is important. Employers should provide training to workers so they understand what heat stress is, how it affects their health and safety, and how it can be prevented (Webb, 2010).

\section{Types of Heat Stress}

\section{1-Heat Stroke}

Heat stroke is the most serious heatrelated disorder. It occurs when the body 
becomes unable to control its temperature: the body's temperature rises rapidly, the sweating mechanism fails, and the body is unable to cool down. When heat stroke occurs, the body temperature can rise to 106 degrees Fahrenheit or higher within 10 to 15 minutes. Heat stroke can cause death or permanent disability if emergency treatment is not given (OSHA, 2011).

Symptoms of heat stroke include: Hot, dry skin (no sweating), hallucinations, chills, throbbing headache ,high body temperature ,confusion/dizziness ,slurred speech.

First Aid: Take the following steps to treat a worker with heat stroke:

- Move the sick worker to a cool shaded area,

- Cool the worker using methods such as: soaking their clothes with water, spraying, sponging, or showering them with water and fanning their body.

\section{2-Heat Exhaustion}

Heat exhaustion is the body's response to an excessive loss of the water and salt, usually through excessive sweating. Workers most prone to heat exhaustion are those that are elderly, have high blood pressure, and those working in a hot environment.
Symptoms of heat exhaustion include: heavy sweating, extreme weakness or fatigue ,dizziness, confusion ,nausea ,clammy, moist skin ,pale or flushed complexion ,muscle cramps ,slightly elevated body temperature, fast and shallow breathing.

First Aid: Treat a worker suffering from heat exhaustion with the following: Take rest in a cool, shaded or air-conditioned area, drink plenty of water or other cool beverages, and take a cool shower, bath, or sponge bath. http://www.webmd.com/ fitness-exercise/heat-exhaustion

\section{3-Heat Syncope}

Heat syncope is a fainting (syncope) episode or dizziness that usually occurs with prolonged standing or sudden rising from a sitting or lying position. Factors that may contribute to heat syncope include dehydration and lack of acclimatization.

Symptoms of heat syncope include: light-headedness, dizziness and fainting

First Aid: Sit or lie down in a cool place when they begin to feel symptoms, slowly drink water, clear juice, or a sports average. https://www.emsaonline.com/mediacenter/ articles/00000164.html

\section{4-Heat Cramps}

Heat cramps usually affect workers who sweat a lot during strenuous activity. 
This sweating depletes the body's salt and moisture levels. Low salt levels in muscles causes painful cramps. Heat cramps may also be a symptom of heat exhaustion.

Symptoms of heat cramps include muscle pain or spasms usually in the abdomen, arms, or legs.

First Aid: Stop all activity, and sit in a cool place, drink clear juice or a sports beverage,don't return to strenuous work for a few hours after the cramps subside because further exertion may lead to heat exhaustion or heat stroke.

Seek medical attention if any of the following apply: the worker has heart problems, is on a low-sodium diet or the cramps do not subside within one hour. http://www.medicinenet.com/heat_cramps/ article.htm

\section{5-Heat Rash}

Heat rash is a skin irritation caused by excessive sweating during hot, humid weather.

Symptoms of heat rash include: heat rash looks like a red cluster of pimples or small blisters, It is more likely to occur on the neck and upper chest, in the groin, under the breasts, and in elbow creases.

First Aid: try to work in a cooler, less humid environment when possible, keep the affected area dry, dusting powder may be used to increase comfort (NIOSH, 2010).

\section{6-Fainting}

A worker who is not accustomed to hot environments and who stands erect and immobile in high temperature may faint due to vasodilatation of the blood vessels of the skin and the lower part of the body in attempts to control internal temperature. Blood will be pooled their rather than return to the heart to be pumped to the brain.

First Aid: Upon lying down, the worker should soon recover. By moving around, and thereby preventing blood from pooling, the patient can prevent further fainting. http://www.webmd.com/skin-problemsand-treatments/tc/heat-rash-topic-overview

\section{7-Transient Heat Fatigue}

Transient heat fatigue refers to the temporary state of discomfort and mental or psychologic strain arising from prolonged heat exposure. Workers unaccustomed to the heat are particularly susceptible and can suffer, to varying degrees, a decline in task performance, coordination, alertness, and vigilance. The severity of transient heat fatigue will be lessened by a period of gradual adjustment to the hot environment (heat acclimatization) (OSHA, 2011).

\section{8-Natural ultraviolet radiation}

Excessive exposure to ultraviolet radiation from sunlight can cause damage 
to the skin and eyes. All skin types can be affected. The level of exposure a person receives can depend upon environmental factors such as season of the year, time of day, cloud cover, and extent of shade and reflection. Clothing, hats, sunscreens and sunglasses also have an effect on level of exposure. In Australia the intensity of the sun at any time of the year in any part of the country can be enough to cause skin cancer.

In the work environment, the amount of exposure to sunlight depends upon time spent outdoors, time of day when tasks are performed outdoors, amount of shade provided to the work area, presence of reflective surfaces in the work area and use of photosensitising chemicals (Webb, 2010).

\section{9-Seasonal heat}

The risk of heat stress arises from a combination of human physical effort, which increases body temperature, and the ability of the body to shed the excess heat in order to maintain a viable core body temperature. Heat loss from the body depends on environmental factors such as radiant heat, air temperature, relative humidity and air movement. It also depends on personal factors such as intensity of physical work, acclimatisation, fitness and state of health, and clothing being worn. Even at rest, the body produces some heat.
Heat loss can occur through conduction, radiation, convection and evaporation of sweat. This last mechanism is the most important in hot conditions (Webb, 2010).

What Occupational control measures can be used to reduce the effects of heat?

The risk of heat-related illnesses can be reduced by:

- Engineering controls to provide a cooler workplace

- Personal Protection to reduce worker exposure

- Training employees to recognize and prevent heat illnesses (Health education).

\section{Engineering Controls}

Engineering controls are the most effective means of reducing excessive heat exposure. The examples which follow illustrate some engineering approaches to reducing heat exposure.

- Reducing Metabolic Heat Production (heat produced by the body): Automation and mechanization of tasks minimize the need for heavy physical work and the resulting buildup of body heat.

- Reducing the Radiant Heat Emission from Hot Surfaces: Covering hot surfaces with sheets of low emissivity material such as aluminium or paint 
that reduces the amount of heat radiated from this hot surface into the workplace.

- Insulating Hot Surfaces: Insulation reduces the heat exchange between the source of heat and the work environment.

- Shielding: Shields stop radiated heat from reaching work stations. Two types of shields can be used. Stainless steel, aluminium or other bright metal surfaces reflect heat back towards the source. Absorbent shields, such as a watercooled jackets made of black-surfaced aluminum, can effectively absorb and carry away heat.

- Ventilation and Air Conditioning: Ventilation, localized air conditioning, and cooled observation booths are commonly used to provide cool work stations. Cooled observation booths allow workers to cool down after brief periods of intense heat exposure while still allowing them to monitor equipment.

- Reducing the Humidity: Air conditioning, dehumidification, and elimination of open hot water baths, drains, and leaky steam valves help reduce humidity.

\section{Personal Protection}

Ordinary clothing provides some protection from heat radiated by surrounding hot surfaces. Specially designed heat- protective clothing is available for working in extremely hot conditions. In hot and humid workplaces, light clothing allows maximum skin exposure and efficient body cooling by sweat evaporation.

Workers who move back and forth between very hot, dry indoor environments and cold winter outdoor environments find that long underwear moderates the extremes in temperatures.

Eye protection which absorbs radiation is needed when the work involves very hot objects, such as molten metals and hot ovens.

Work that requires the wearing of impermeable clothing presents an added heat burden as the clothing reduces the body's ability to dissipate heat. Under such circumstances, it is often necessary to reduce the exposure limit values to levels below those appropriate for workers wearing light clothing, (http://www.bls. gov/oco/ocos226.htm).

\section{Can we become acclimatized to hot environments?}

The body adapts to a new thermal environment by a process called acclimatization. Complete heat acclimatization generally takes six to seven days, but some individuals may need longer. Loss of acclimatization occurs gradually when a person is moved permanently 
away from a hot environment. However, a decrease in heat tolerance occurs even after a long weekend. As a result of reduced heat tolerance, it is often not advisable for anyone to work under very hot conditions on the first day of the week.

New employees should acclimatize before assuming a full workload. It is advisable to assign about half of the normal workload to a new employee on the first day of work and gradually increased on subsequent days.

Although well-trained, physically fit workers tolerate heat better than people in poor physical condition, fitness and training do not substitute for acclimatization.

Some medications interfere with acclimatization. For example, hypotensives, diuretics, antispasmodics, sedatives, tranquilizers, antidepressants and amphetamines decrease the body's ability to cope with heat. Workers should seek a doctor's advice on the suitability of a medication for them if they work in hot environments. Consumption of alcohol also interferes with acclimatization (NIOSH, 2010b).

\section{How can we prevent heat related illnesses?}

If practical, workers in hot environments should be encouraged to set their own work and rest schedules. Infrequent or irregular tasks such as emergency repairs of hot process equipment often result in heat exposure. Experienced workers can often judge heat strain and limit their exposure accordingly. Inexperienced workers may need special attention as they may continue to work beyond the point at which signs of heat strain appear.

People are generally unable to notice their own heat stress related symptoms. Their survival depends on their co-worker's ability to recognize these symptoms and seek timely first aid and medical help.

Salt and Fluid Supplements: A person working in hot environment loses water and salt through sweat. This loss should be compensated by water and salt intake. Fluid intake should equal fluid loss. On average, about one litre of water each hour may be required to replace the fluid loss. Plenty of cool $\left(10-15^{\circ} \mathrm{C}\right)$ drinking water should be available on the job site and workers should be encouraged to drink water every 15 to 20 minutes even if they do not feel thirsty.

An acclimatized worker loses relatively little salt in their sweat and, therefore, the salt in the normal diet is usually sufficient to maintain the electrolyte balance in the body fluids. For unacclimatized workers who may sweat continuously and repeatedly, additional salt in the food may be used. Salt tablets are not recommended because the salt does not enter the body system 
as fast as water or other fluids. Too much salt can cause higher body temperatures, increased thirst and nausea. Workers on salt-restricted diets should discuss the need for supplementary salt with their doctor (ACGIH, 2012).

Sport drinks, fruit juice, etc: Drinks specially designed to replace body fluids and electrolytes may be taken but for most people, they should be used in moderation. They may be of benefit for workers who have very physically active occupations but keep in mind they may add unnecessary sugar or salt to your diet. Fruit juice or sport and electrolyte drinks, diluted to half the strength with water, is an option. Drinks with alcohol or caffeine should never be taken, as they dehydrate the body. For most people, water is the most efficient fluid for re-hydration.

Emergency Action Plan: In extreme environments, an emergency plan is needed. The plan should include procedures for providing affected workers with first aid and medical care http://www.bls.gov/oco/ ocos 226. htm.

\section{References}

1. American Council of Governmental Industrial Hygienists (ACGIH) (2012) :TLV for Chemical Substances and Physical Agents and Biological Exposure Indices.

2. American Conference of Governmental Industrial Hygienists (ACGIH) (2008):
Threshold Limit Values for Chemical Substances and Physical Agents and Biological Exposure Indices p. 221.

3. American Conference of Governmental Industrial Hygienists (2006) : TLVs \& BEIs, Based on the Documentation of the Threshold Limit Values for Chemical Substances and Physical Agents \& Biological Exposure Indices. Also known as, "the TLV booklet."

4. Harrington JM (2001): Health effects of shift work and extended hours of work. Occup Environ Med; 58:68-72 .

5. NIOSH (2010)a: Occupational exposure to hot environments .On line on www.osha.com.

6. NIOSH (2010)b : Safety and Health Topic: Heat Stress. Available on www.niosh.com.

7. OSHA (2011): Protecting workers from heat stress. [Online]. Available on: http://www.osha. gov/Publications/osha3154.

8. OSHA (2010): Hot Environments - Control Measures. In: Canadian Centre for Occupational Health \& Safety; on line on www.osha.com.

9. Rodahl K (2003): Occupational health conditions in extreme environments. Ann Occup Hyg. ; 47 (3):241-52.

10. Webb G (2010) : Outdoor Workers Exposed To Ultraviolet Radiation \& Seasonal Heat.In: The University of Sydney. Australia. Available on www.niosh.com.

11. Bureau of Labor Statistics, U.S. Department of Labor, Occupational Outlook Handbook, 201011 Edition, Welding, Soldering, and Brazing Workers, on the Internet at http://www.bls.gov/oco/ocos226.htm https://www.emsaonline.com/mediacenter/ articles/00000164.html

http://www.medicinenet.com/heat_cramps/article. htm

http://www.webmd.com/fitness-exercise/heatexhaustion

http://www.webmd.com/skin-problems-andtreatments/tc/heat-rash-topic-overview 